\title{
AN AUTOMATED ULTRASONIC SYSTEM FOR INSPECTION OF AIRCRAFT TURBINE ENGINE COMPONENTS
}

\author{
D. Stubbs, I. Fiscus, D. Gasper, G. Hartman, J. Hoeffel, W. Hoppe, V. Kramb, S. Kuhlman, R. Martin, R. Olding, \\ D. Petricola, N. Powar, J. Sebastian \\ University of Dayton Research Institute, Dayton, OH USA
}

\begin{abstract}
The University of Dayton Research Institute (UDRI), under contract by the U.S. Air Force, has designed and constructed a fully automated ultrasonic inspection system for the detection of embedded defects in rotating gas turbine engine components. This system uses many new approaches to conducting automated ultrasonic inspections to produce engine component accept/reject decisions that can be based on probability of detection (POD) information without human intervention. The ultrasonic inspection system incorporates off-the-shelf, industry standard components typically not found in NDI systems, which have been integrated using a modulararchitecture approach, to produce inspections of engine components in an Air Force depot environment. Designed to be rugged and easily maintained, the system consists of a six-axis articulated robotic arm, rotating water tank, phased-array ultrasonic instrument, automated probe changer, "smart" probes, collision avoidance hardware, PCbased industrial computer, Windows-based operating system, digital vision system, and a specialized water management system to eliminate water bubbles. Use of the phased-array ultrasonic instrument and probes allows for optimization of both the sensitivity and resolution for each inspection through electronic beamforming, scanning, and focusing processes. The system requires an operator to load the part, and then conducts all parts of the inspection automatically, including part recognition, part dimensioning, probe changing, calibration, inspection, and accept/reject decisions. This paper discusses, in detail, specifications of the system and technology improvements that have been incorporated into the system.
\end{abstract}

Introduction: A traditional, $\mathrm{X}, \mathrm{Y}, \mathrm{Z}$ immersion $\mathrm{C}$-scan system is shown in Figure 1. This system is similar to most traditional ultrasonic immersion $\mathrm{C}$-scan systems that consist of the following components:

- $\quad$ A motorized, mechanical manipulator for moving the ultrasonic probe

- $\quad$ Controllers and power equipment for the manipulator motors

- $\quad$ A tank of water

- $\quad$ A water management system

- $\quad$ An ultrasonic instrument

- $\quad$ Ultrasonic transducers - manually attached

- $\quad$ A turntable with test object fixturing

- $\quad$ A data acquisition computer

- Data acquisition and processing software

Improvements are constantly being made in all of these components, but the basic system architecture has remained the same for several decades. The mechanical manipulator sits on top of a (usually) rectangular water tank and moves about the tank in a rectangular coordinate system. Movement of the ultrasonic probe is commonly done in a plane, and some advanced systems can move the transducer along nonlinear paths. A secondary manipulator is often used to provider two orthogonal probe angulation axes - sometimes referred to as gimbal/gimbal or gimbal/swivel axes.

The water management system circulates the water in the tank to filter it and keep it clean. Water is added to the tank as needed large additions of water often take the system out of use, for up to 24 hours, to allow air bubbles leave the water.

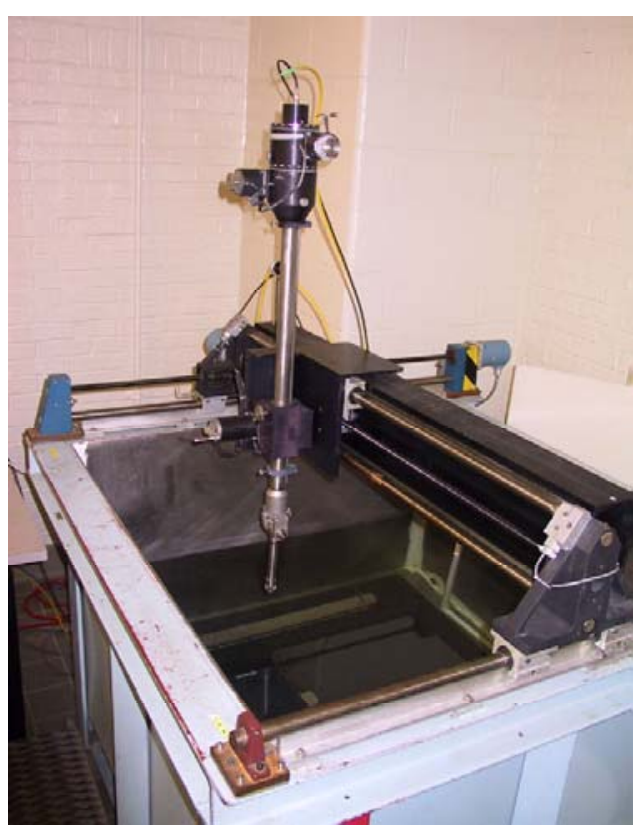

Figure 1 -Traditional ultrasonic C-scan svstem

The traditional ultrasonic instrument has a pulser that is either a spike type, or the newer square-wave type. Higher priced instruments include depth-amplitude correction capability and broadband receivers with $80 \mathrm{~dB}$ of adjustable gain. Transducer interfaces allow pulse-echo and through-transmission usage and some instruments can accept four, eight, or even sixteen transducers. The transducers used in these systems contain single piezoelectric elements and often use a lens for focusing. The connection of the transducer to the mechanical manipulator is 
usually through a "search tube" - a precisely machined metal cylinder containing electrical wires and connectors that extends the position of the transducer away from the manipulator.

In ultrasonic systems used to inspect circular or cylindrical components, a turntable is placed in the water in a position such that the mechanical manipulator can position the transducer over the test object on the turntable. The turntable holds a multi-jaw chuck that forces the test object to be centered over the turntable axis. The turntable motor, depending on the particular design, is submersed, or placed above the water and coupled with shafts/belts to the turntable, or is positioned under the tank and connects to the turntable through watertight bearings.

In recent years ultrasonic system manufacturers have integrated PC hardware and software into their systems. Pulser-receiver cards and digitizers are available that fit on standard PC buses. Data acquisition and signal/image processing often use software running on standard PC operating systems. Motion control is done by defining manipulator scanning/indexing step sizes and ranges. Data acquisition occurs by synchronizing the digitizer with the motion steps or the pulser-receiver trigger.

Improvements to ultrasonic systems have been made over the years, usually through incremental upgrades of the various system components. Even so, today's state-of-the-art immersion ultrasonic C-scan system is similar to systems from 20 or 30 years ago.

Results: Many technologies developed for other industries are now available to the NDT systems industry. However, the introduction of new technologies to different applications can be expensive and risky and often occurs only when backed by national R\&D or military budgets. In 2001 the University of Dayton Research Institute (UDRI) in Dayton, Ohio was asked by the U.S. Air Force to design a new immersion ultrasonic inspection system for use on turbine engine components. This system was to be automatic, i.e., no human interaction with the inspection process. Also, UDRI was given a blank slate for the design; the goal was to produce a design optimized for the inspection of engine components and be suitable for a depot environment.

UDRI designed a new automated ultrasonic inspection system that used many new technologies developed for other industries. After the nine month design effort UDRI built a prototype system to demonstrate the feasibility of using the new technologies in an ultrasonic NDT system. System components generally were off-the-shelf, mature, and selected from vendors expected to able to supply the components for many years. Some of the new components of the system are:

- A six-axis robot as the mechanical manipulator

- An open architecture robot controller that incorporated movement accuracy improvements

- Automated transducer changing

- A cylindrical, acrylic water tank

- A turntable that supported and rotated the entire water tank

- No part fixturing

- A water control system that fills/empties the tank for each inspection

- A 128 channel, phased array ultrasonic instrument

- Phased array transducers with up to 128 elements

- An integrated digital vision system

- Standard industrial PCs running Windows XP and C.net software

- A graphical user interface for designing inspections ("point and click")

\section{Discussion:}

Six-axis Robotic Manipulator and Enhanced Controller

Industrial robots have many advantages over traditional $\mathrm{X}, \mathrm{Y}, \mathrm{Z}$ gantry systems for use as the transducer manipulator in ultrasonic systems.

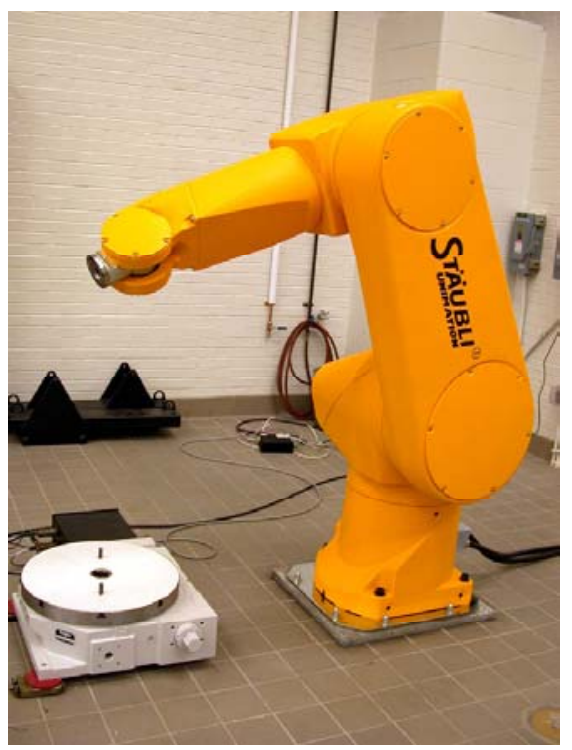

Figure 2 - Six-axis robot used in the new ultrasonic system 
Table 1 lists important characteristics of ultrasonic system mechanical manipulators with comparisons between traditional gantry style manipulators, a standard six-axis industrial robot, and the same six-axis robot with UDRI developed enhancements. The robot used by UDRI is shown in Figure 2.

Table 1 - Characteristics of Gantry Style and Six-axis Robotic Mechanical Manipulators

\begin{tabular}{|c|c|c|c|}
\hline Characteristic & $\begin{array}{l}\text { X,Y,Z Gantry System } \\
\text { with A,B Gimbal }\end{array}$ & $\begin{array}{l}\text { Standard Six-axis } \\
\text { Industrial Robot }\end{array}$ & $\begin{array}{c}\text { Standard Six-axis } \\
\text { Industrial Robot with } \\
\text { UDRI Improvements }\end{array}$ \\
\hline Positional Repeatability & $0.05 \mathrm{~mm}$ (0.002 inches) & $0.05 \mathrm{~mm}$ (0.002 inches) & $0.05 \mathrm{~mm}$ (0.002 inches) \\
\hline Positional Accuracy & $\begin{array}{l}0.05 \mathrm{~mm} \text { per } 300 \mathrm{~mm} \\
(0.002 \text { inches per foot })\end{array}$ & $\begin{array}{l}1 \mathrm{~mm} \text { per } 300 \mathrm{~mm} \\
(0.04 \text { inches per foot })\end{array}$ & $\begin{array}{l}0.05 \mathrm{~mm} \text { per } 300 \mathrm{~mm} \\
(0.002 \text { inches per foot })\end{array}$ \\
\hline Transducer Orientation & $\begin{array}{l}\text { Spherical coordinates at } \\
\text { end of "manipulator" }\end{array}$ & $\begin{array}{l}\text { Any orientation, any } \\
\text { location }\end{array}$ & $\begin{array}{l}\text { Any orientation, any } \\
\text { location }\end{array}$ \\
\hline Movement Speed & $\begin{array}{l}250 \mathrm{~mm} / \mathrm{sec} . \\
(10 \mathrm{in} . / \mathrm{sec} .) \\
\end{array}$ & $\begin{array}{l}2000 \mathrm{~mm} / \mathrm{sec} . \\
(80 \mathrm{in} . / \mathrm{sec} .)\end{array}$ & $\begin{array}{l}2000 \mathrm{~mm} / \mathrm{sec} . \\
(100 \mathrm{in} . / \mathrm{sec} .)\end{array}$ \\
\hline Mean Time Between Failure & varies & 50,000 hours & 50,000 hours \\
\hline Maintenance & $\begin{array}{l}\text { Replacement of } \\
\text { bearings; rail alignment }\end{array}$ & $\begin{array}{l}\text { Change oil and battery } \\
\text { annually }\end{array}$ & $\begin{array}{l}\text { Change oil and battery } \\
\text { annually }\end{array}$ \\
\hline Ease of Control & Straight-forward & Complicated & Graphical User Interface \\
\hline Payload Capacity & Low - $1 \mathrm{~kg}(2.2 \mathrm{lbs})$. & $25 \mathrm{~kg}$ (55 lbs.) & $25 \mathrm{~kg}$ (55 lbs.) \\
\hline Cost & $\$ 35,000$ to $\$ 200,000$ & $\$ 37,000$ to $\$ 75,000$ & $\$ 42,000$ to $\$ 80,000$ \\
\hline Ease of Expansion & $\begin{array}{l}\text { Difficult - entire tank, } \\
\text { manipulator changes }\end{array}$ & $\begin{array}{l}\text { Select next larger robot } \\
\text { model }\end{array}$ & $\begin{array}{l}\text { Select next larger robot } \\
\text { model }\end{array}$ \\
\hline Auto. Transducer Changing & Very difficult & $\begin{array}{l}\text { Industry standard } \\
\text { hardware }\end{array}$ & $\begin{array}{l}\text { Industry standard } \\
\text { hardware }\end{array}$ \\
\hline
\end{tabular}

The most important improvement UDRI and its team member American Robot Corporation, have made to the standard industrial six-axis robot is the improved accuracy of transducer location to be less than $0.25 \mathrm{~mm}$ per meter of travel. The accuracy improvements are possible through one-time use of commercially available optical measurement systems along with appropriate error corrections in the robot controller.

The high positional accuracy is maintained beyond the end of the robot arm all the way to the face of the ultrasonic transducer. This is accomplished by storing dimensional information unique to each probe/transducer assembly in a memory chip in the transducer housing. When the robot picks up the probe, the dimensional data is sent to the robot controller which incorporates the exact dimensions of the transducer into all movements.

\section{Cylindrical Rotating Water Tank}

Because the industrial robot is very flexible in manipulating the transducer and supports automated probe changing, the immersion tank does not have to be physically connected to the mechanical manipulator. This allows the choice of a water tank that "fits" the test objects - in this case cylindrical engine disks. A commercial source of large diameter, cylindrical, acrylic fish aquariums is used. These cylinders can be ordered in a wide range of diameters (up to 2.3 meters) and cut to any desired length. A photograph of a $1.05 \mathrm{~m}$ diameter acrylic tank used on the UDRI ultrasonic system is shown in Figure 3.

By limiting the amount of water in the tank (due to the smaller volume than traditional systems) and separating the tank from the mechanical manipulator, new approaches to using turntables were possible. UDRI settled on choosing an industry-standard, precision turntable that could rotate the entire water tank. With a moderate size test object, and the tank filled with water (about 600 liters), the total mass rotating is less than 800 $\mathrm{kg}$. Testing has shown that an immersed $300 \mathrm{~kg}$ object can be rotated at 25 r.p.m. Accuracy is $\sim 0.005$ degrees.

Another interesting design feature is the placement of an X-Y table between the turntable and the water tank. Through use of a video camera (described in a later section) the test object can be centered on the turntable through X-Y adjustments of the entire tank assembly. Centering accuracies of $0.05 \mathrm{~mm}$ are 
achieved. This does mean that the water tank "oscillates" when rotated, but only within the X-Y centering adjustment limits allowed (typically $12 \mathrm{~mm}$ ). The oscillation, typical less than $12 \mathrm{~mm}$ is barely noticeable when watching the one meter diameter water tank rotate.

In normal use the turntable rotates at less than one r.p.m. because of the very high ultrasonic scanning rates achieved using the phased array instrumentation. The relatively low rotation speeds, compared to traditional systems, eliminates the need to clamp the test object.

Significant improvements are gained by not submersing the turntable, providing automated test object centering, and not requiring clamping fixtures. These improvements are expected to be well received by engine maintenance/inspection depots and industrial inspection houses.

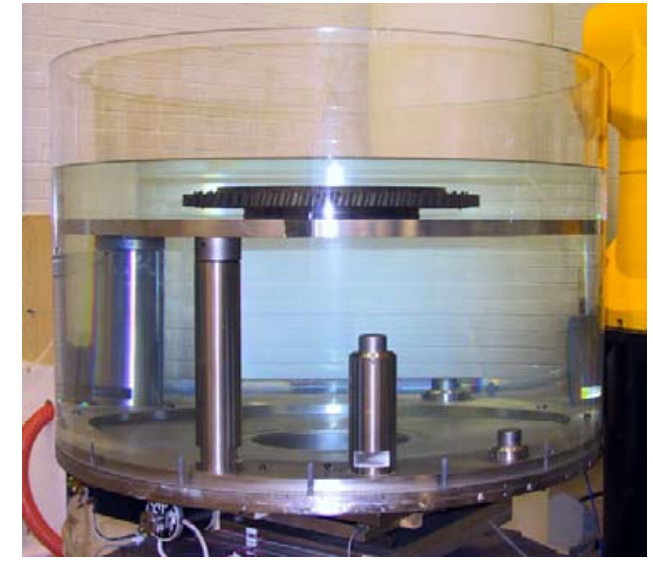

Figure 3 - Cylindrical water tank mounted on the system turntable

\section{Water Management System}

UDRI incorporated several new features in the water management for the system. To accommodate the vision system the water level in the tank must be lowered to below the surface of the test object. Then, for ultrasonic inspections the water level is raised above the test object. Control of water level is straight-forward using readily available pumps, valves, level sensors, etc. However, it is a rule of thumb in immersion ultrasonic NDT that water tanks are left to "deaerate" for 24 hours after filling with water - and this is written into specifications for some inspections.

UDRI has developed a very simple method for removing air bubbles from the water that is pumped into and out of the inspection tank. By continuously mechanically dispersing the water in a storage tank, clear, bubblefree water is pumped into the inspection tank and remains bubble free indefinitely. The dispersal process, which really is simply spraying the water through a nozzle, would be expected to add air to the water resulting in more bubbles in the water. However, just the opposite has been found to occur.

\section{Phased Array Ultrasonic Probes and Instrumentation}

Phased array ultrasonic instruments and probes are used extensively in the medical community, but are relatively new to the industrial NDT community. For NDT use the instrumentation is expensive and complicated to set up. The probes are more difficult to design and choose for a given inspection. However, the added value that phased array instrumentation brings to an inspection is significant. Ultrasonic beams can be focused at different depths using the same transducer. Electronic scanning of the beam is fast and accurate. Beam steering is possible and allows inspection of curved surfaces quickly and precisely.

UDRI has chosen $5 \mathrm{MHz}$ and $10 \mathrm{MHz}$ transducers containing 128 elements for use in this system (see Figure 4). The array lengths (from element \#1 to \#128) are $96 \mathrm{~mm}$ and $76 \mathrm{~mm}$ respectively with up to 32 elements active at any given time. Thus, test objects - as an example, turbine engine components having bores up to $75 \mathrm{~mm}$ high - can be inspected without indexing the transducer across the object. The array probe electronically moves the ultrasonic beam (formed using 32 consecutive elements) from element \#1 to element \#96 which effectively moves the ultrasonic beam up to $3 / 4$ of the transducer width in less than one second. Thus, complete inspection of an engine component bore can be done with only one rotation of the turntable.

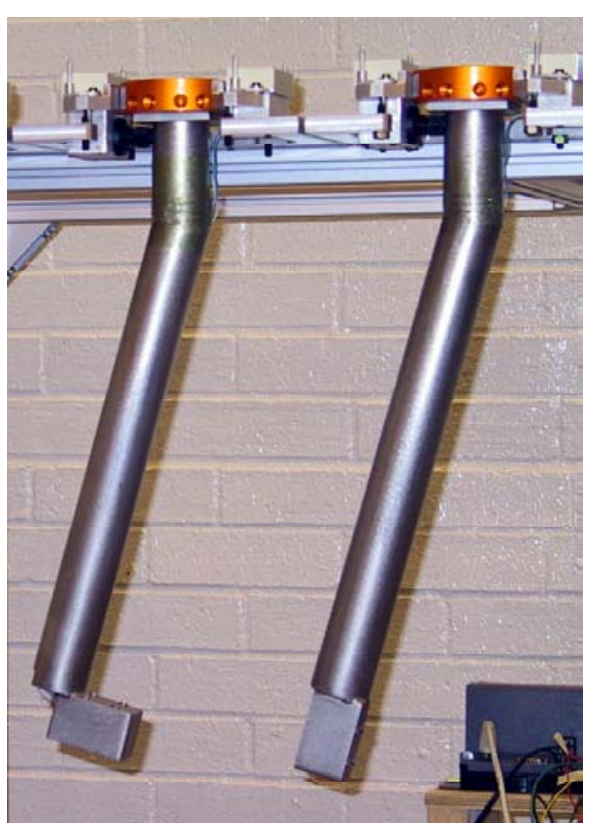

Figure 4 - Phased array ultrasonic probes hanging in the probe 
The phased array probes also can be focused at different depths within the test object removing the need to change transducers. The electronic focusing can be done "on the fly" so that a given "slice" (B-scan) of a test object can be inspected with ultrasonic beams focused at different depths along the slice. The multiple interrogations at different depths of a given slice takes more time, but can still be done in just seconds. More information about the use of phased array probes can be found in these proceedings. The inspection that results from use of the phased array instrument and probes is quite different than a traditional C-scan of a circular part. Instead of having the transducer index down through the part while the turntable makes dozens or hundreds of high speed rotations, the phased array inspection occurs with the turntable making one or two rotations at speeds of, for example 0.2 r.p.m.

\section{Collision Avoidance Technologies}

Several features have been built into the system to avoid damaging collisions between the robot and other objects. The first feature is based upon defining separate areas of movement, or "work cells" in the system. Several work cells are defined: the water tank, the calibration tank, and the probe pickup station. The software to guide the movements of the robot can be changed only for movements within an individual work cell. The software that controls movements between work cells is not open to change. Thus, an inspection designer can move the probe anywhere desired within the inspection tank, but cannot move the probe beyond the limits of the tank. The limitations on range of movements combined with the use of thoroughly tested software for cell-to-cell movement helps reduce collisions.

A second collision avoidance feature is the use of a digital vision system to survey the inspection tank at the beginning of each inspection. The "view" of the inspection tank must match the expected view stored in the system computer's memory or the inspection is aborted. More information on the use of the digital vision system is contained in the next section.

A third collision avoidance mechanism is the incorporation of a commercially available "break-away clutch" between the end of the robot arm and the probe connector. This clutch disengages if the probe encounters forces in any direction and automatically stops the robot arm motion. The disabling force can be adjusted during an inspection to provide very sensitive collision detection while the probe is near an engine component, and less sensitivity when the probe is being quickly moved along "known clear paths" between work cells. After the clutch "breaks away" it can be reconnected with an alignment accuracy of better than $0.025 \mathrm{~mm}$.

\section{Integrated Digital Vision System}

The new automated ultrasonic inspection system uses a digital camera to:

- Check for the correct test object

- Check for the correct side of the object

- Center the test object on the turntable

- Rotate the turntable such that the " 0 degree" location of the test object is at a known position.

These checks and measurements are critical to the safe functioning of an automated system. The system must protect itself, and the test object, from damage due to collisions - an almost certainty if an incorrect test object is accidentally placed in the system.

UDRI has chosen an industrial 4 megapixel digital camera for the system. The camera is picked up by the robot in the same way as are ultrasonic probes and then moved over the inspection tank (Figure 5). The camera is positioned about one meter above the engine component and several images are captured and then processed with a commercial image processing package. Image processing routines are used to determine if the engine part that is loaded is the correct part to be inspected, if the proper side of the engine part is facing upward, and

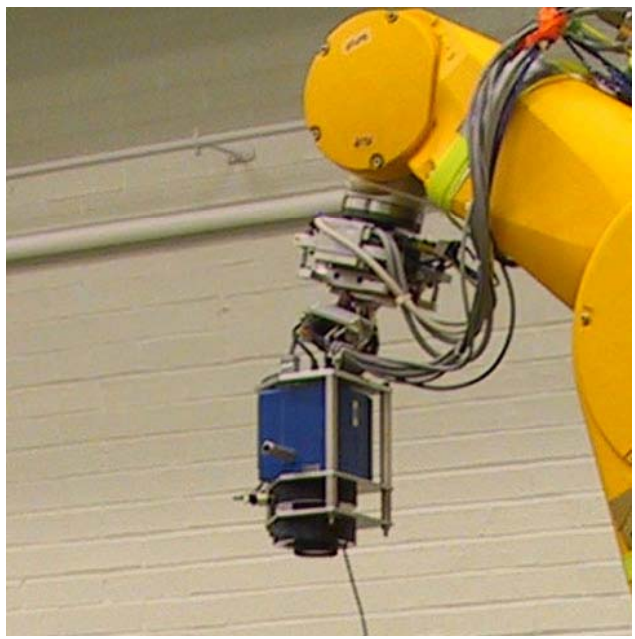

Figure 5 - Digital camera picked up by the robot to image the test object

how much the engine part is off-center relative to the axis of the turntable. Centering of the engine part is automatically done by moving the entire water tank on an X-Y table that is positioned between the tank and the turntable. Centering accuracies of $0.075 \mathrm{~mm}$ are achievable. 
Control of the illumination of the engine component is very important for reliable image processing. The system incorporates a commercially available, long working distance, ring light that illuminates a $500 \mathrm{~mm}$ diameter circle at the plane of the engine component.

Testing has shown that the digital vision system has approximately $0.1 \mathrm{~mm}$ resolution over a field of view of $500 \mathrm{~mm}$ - achieved using subpixel interpolation. Test object recognition, part centering on the turntable, and circumferential alignment of the engine part takes approximately 50 seconds.

\section{Graphical User Interface}

Modern PC hardware and software allows the inclusion of inspection tools that rely heavily on graphical users interfaces. For example, "scan plans", the test-object-specific software that controls an inspection, are created by pointing and clicking with a PC mouse to desired features on a cross-sectional representation of the test object. Instead of writing lines of code to direct the scanning and indexing of the ultrasonic transducer, mouse clicks select test object surfaces defining the beginning and ending of scans. Appropriate robot commands are automatically generated by the PC to be sent to the robot controller at the time of inspection.

Similarly, the inspection results made available to the system operator are graphically based. Representations (pseudo 3D) of the test object are shown to the operator at the end of the

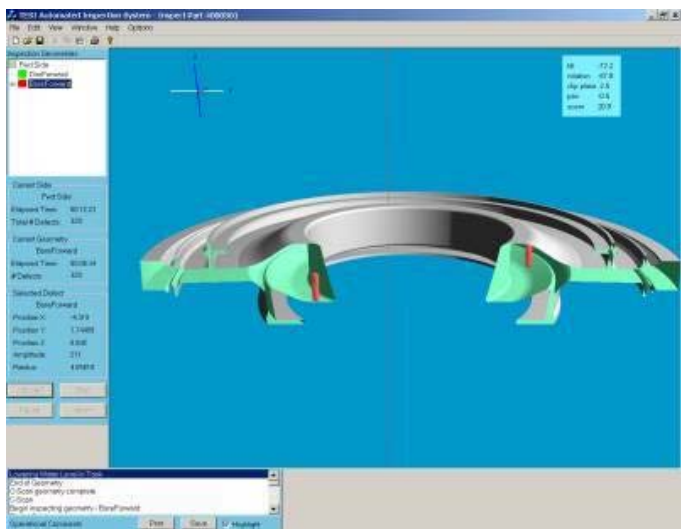

Figure 6 - Graphical representation of defects in a 3D view of the test object inspection with the locations of defects accurately placed within the object. Thus, operators quickly and easily see exact locations of the defects relative to the features of the test object (see Figure 6).

\section{Evaluation of System Sensitivity and Probability of Detection (POD)}

The immersion ultrasonic NDT community generally quantifies the sensitivity of an inspection system by specifying the amplitude of response from a flat bottom hole (FBH). Using FBHs has some advantages - they are familiar to practitioners in the UT NDT community, are commercially available, and give assurance that an embedded defect producing that level of response must have a cross-sectional dimension equal to or larger than the referenced FBH diameter.

Some of the disadvantages of using FBHs to quantify the sensitivity of an ultrasonic system are that a given hole is useful for only one mode of ultrasonic propagation, small diameter holes are difficult to make deep in metal, and variations in signal amplitude can occur from holes that are nominally the same size. Also, FBHs are not very representative of the types of defects that are usually found in test objects.

UDRI has begun tasks to take a step to produce embedded defects in engine alloys that overcome some of the disadvantages listed above. The defects are commercially available spheres, with a diameter of $3 \mathrm{~mm}$, that have been embedded in Ti-6-4 and Rene 95.

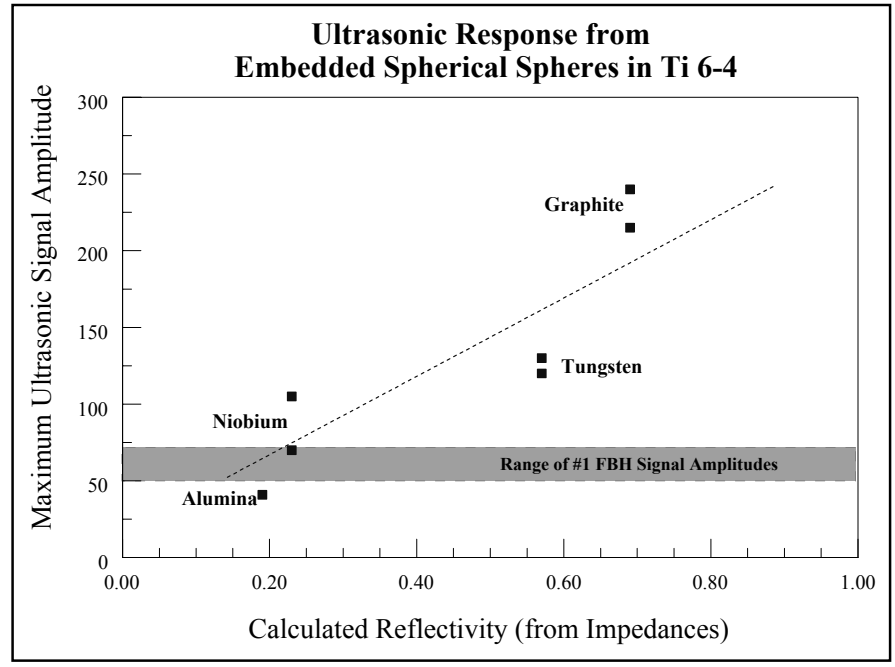

Figure 7 - Ultrasonic signal responses from embedded sphere specimens 
Spheres of different materials can be obtained, which give differing reflectivities in the engine alloys. For this work spheres made of graphite, $\mathrm{Al} 2 \mathrm{O} 3, \mathrm{Nb}$, and $\mathrm{W}$ have been used. Careful processing of the alloy assemblies eliminates any bond lines in the specimens.

Figure 7 shows ultrasonic data from the embedded spheres. The amplitudes of reflection from the embedded spheres are plotted against the calculated reflectivity in Ti-6-4. It is important to note that the amplitude of the reflected signals spans a range from less than a \#1 FBH to almost that expected from a \#2 FBH. Varying the size and composition of the spheres will allow specimens to be made that produce signals that span a large range; ultimately POD data may be available for spherical defects as a function of size and composition.

Conclusion: A new, unique, automated ultrasonic inspection system (see Figure 8) has been designed that incorporates new, off-the-shelf technologies and components. These components have been developed for other applications but can be integrated into NDT systems. Significant advantages in inspection speeds and sensitivity result from bringing these technologies together in an integrated system. The integrated components mutually support each other resulting in a system that offers more benefits than the sum of the individual components would suggest. Many of the technologies tested and demonstrated in this ultrasonic system can be used in other NDT systems. The wide range of technologies currently available due to advances in the microprocessor, electronics, robotics, and materials industries are ready to be incorporated into NDT systems and can result in large leaps

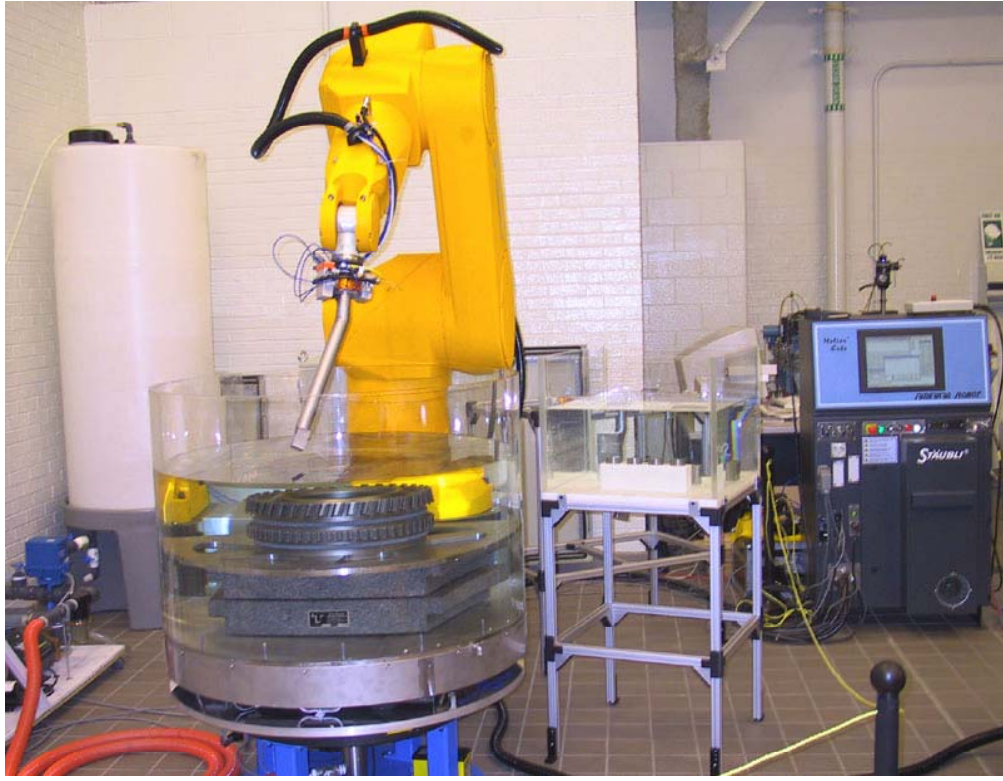

Figure 8 - The new Automated Ultrasonic Inspection System forward in inspection capabilities.

Acknowledgements: This work was conducted under contract with the U.S. Air Force, contract \#F42620-00-D0039, RZ02. The authors want to acknowledge the contributions to the project given by personnel at American Robot Corporation, General Electric Aircraft Engines, R/D Tech, and U.S. Inspection Services. The authors also want to express their thanks to the engineering students at the University of Dayton who have worked on this program: Justin Spenard, Matt Pacyna, Paul Demmy, Aaron Riesen, Greg Raffio, Greg Nickels, Christy Pastor, Jonathan Jonela, James McMahon, Mike Pateras, and Ben Tricase.

\section{References:}

1. V. Kramb, "Defect Detection and Classification in Aerospace Materials Using Phased Array Ultrasonics", published elsewhere in these proceedings.

2. The spherical embedded defect specimens have been developed under the same program that funded the work described in this paper. The data shown in Figure 7 were obtained from Dr. Jon Bartos, a consultant on the TESI program, working with Dr. Mike Gigliotti and Dr. Bob Gilmore of the GE Global Research Center. 